\title{
Consensus definitions of 14 severe acute toxic effects for childhood lymphoblastic leukaemia treatment: a Delphi consensus
}

\begin{abstract}
Kjeld Schmiegelow, Andishe Attarbaschi, Shlomit Barzilai, Gabriele Escherich, Thomas Leth Frandsen, Christina Halsey, Rachael Hough, Sima Jeha, Motohiro Kato, Der-Cherng Liang, Torben Stamm Mikkelsen, Anja Möricke, Riitta Niinimäki, Caroline Piette, Maria Caterina Putti, Elizabeth Raetz, Lewis B Silverman, Roderick Skinner, Ruta Tuckuviene, Inge van der Sluis, Ester Zapotocka, on behalf of the Ponte di Legno toxicity working group*
\end{abstract}

\begin{abstract}
Although there are high survival rates for children with acute lymphoblastic leukaemia, their outcome is often counterbalanced by the burden of toxic effects. This is because reported frequencies vary widely across studies partly because of diverse definitions of toxic effects. Using the Delphi method, 15 international childhood acute lymphoblastic leukaemia study groups assessed acute lymphoblastic leukaemia protocols to address toxic effects that were to be considered by the Ponte di Legno working group. 14 acute toxic effects (hypersensitivity to asparaginase, hyperlipidaemia, osteonecrosis, asparaginase-associated pancreatitis, arterial hypertension, posterior reversible encephalopathy syndrome, seizures, depressed level of consciousness, methotrexate-related stroke-like syndrome, peripheral neuropathy, high-dose methotrexate-related nephrotoxicity, sinusoidal obstructive syndrome, thromboembolism, and Pneumocystis jirovecii pneumonia) that are serious but too rare to be addressed comprehensively within any single group, or are deemed to need consensus definitions for reliable incidence comparisons, were selected for assessment. Our results showed that none of the protocols addressed all 14 toxic effects, that no two protocols shared identical definitions of all toxic effects, and that no toxic effect definition was shared by all protocols. Using the Delphi method over three face-to-face plenary meetings, consensus definitions were obtained for all 14 toxic effects. In the overall assessment of outcome of acute lymphoblastic leukaemia treatment, these expert opinion-based definitions will allow reliable comparisons of frequencies and severities of acute toxic effects across treatment protocols, and facilitate international research on cause, guidelines for treatment adaptation, preventive strategies, and development of consensus algorithms for reporting on acute lymphoblastic leukaemia treatment.
\end{abstract}

\section{Introduction}

Acute lymphoblastic leukaemia accounts for $25 \%$ of all childhood cancers and has leapt from being universally fatal two generations ago, to having 5 -year overall survival rates of more than $90 \%$ with the best contemporary treatment. ${ }^{1}$ However, a substantial number of patients have severe, fatal, or lifelong toxic effects. ${ }^{2}$ The frequency of these toxic effects varies widely across study protocols (appendix), which reflects not only the difference in treatment intensities, but also the diverse definitions of toxic effects and the strategies for their identification and reporting, making meaningful comparisons of the risks of toxic effects impossible.

The progressive intensification of acute lymphoblastic leukaemia treatment in the past three decades means that the chance of treatment-related death can now be equal to the chance of leukaemic relapse in low-risk patients. ${ }^{3}$ Accordingly, trials no longer aim only to introduce more powerful antileukaemic drugs, but also focus on minimising toxic effects. Evaluation of the success of this approach depends on robust measurement of the toxic effect burden within different groups in a trial, between different trials internationally, and between patient subsets defined by clinical features or germline DNA variants. ${ }^{4}$

Definitions for most organ toxic effects already exist, and the US National Cancer Institute Common
Terminology Criteria for Adverse Events (CTCAE) ${ }^{5}$ is widely used. However, the CTCAE describes many toxic effects in very general terms, and was not developed to meet the specific needs associated with childhood acute lymphoblastic leukaemia treatment. Additionally, the grades of toxic effects that are identified and reported vary across protocols. Finally, the scientific community uses various definitions for several toxic effects (appendix $\mathrm{p}$ 24) and there is a need for consensus definitions across paediatric acute lymphoblastic leukaemia protocols.

Recognising the need for international collaboration on this issue, the Ponte di Legno consortium (PdL) established a toxicity working group (PTWG) to address serious adverse events associated with childhood acute lymphoblastic leukaemia treatment (appendix), and thus improve the outcomes of children with the disease. ${ }^{1}$ As a first step, the PTWG aimed to obtain consensus definitions of 14 prioritised acute toxic effects. We report the process and the final definitions that have been approved by the PdL acute lymphoblastic leukaemia groups. We hope these definitions will be valuable for reliable comparisons of data on toxic effects emerging from various treatments for acute lymphoblastic leukaemia, for collaborative research addressing risk factors including host genome variants, and for strategies for the prevention or treatment of toxic effects.
Lancet Oncol 2016; 17: e231-39 *See appendix for Ponte di Legno toxicity working group members

Department of Pediatrics and Adolescent Medicine, Rigshospitalet, Copenhagen, Denmark

(Prof K Schmiegelow MD T L Frandsen MD); Institute of Clinical Medicine, Faculty of Medicine, University of Copenhagen, Copenhagen, Denmark (Prof K Schmiegelow); Department of Pediatric Oncology, Langone Medical Center, New York University, New York, NY, USA (Prof K Schmiegelow); Department of Pediatric Hematology and Oncology, St Anna Children's Hospital, Vienna, Austria

(A Attarbaschi MD); Department of Pediatric and Adolescent Medicine, Medical University of Vienna, Vienna, Austria (A Attarbaschi); Schneider Children's Medical Center of Israel, Department of Pediatric Hematology Oncology, Petah-Tikva, Israel (S Barzilai MD); University Medical Center Eppendorf, Clinic of Pediatric Hematology and Oncology, Hamburg, Germany (G Escherich MD); Institute of Cancer Sciences, College of Medical, Veterinary and Life Sciences, University of Glasgow, Glasgow, UK (C Halsey MD); University College London's NHS Foundation Trust, London, UK (R Hough MD); St Jude Children's Research Hospital, Memphis, TN, USA (Prof S Jeha MD); Children's Cancer Center, National Center for Child Health and Development, Tokyo, Japan (M Kato MD); Division of Pediatric

Hematology-Oncology, Mackay Memorial Hospital, Taipei, Taiwan (D-C Liang MD); Department of Pediatrics, Aarhus University Hospital, 


\section{Aarhus N, Denmark \\ (TS Mikkelsen MD); \\ Christian-Albrechts-University \\ Kiel and University Medical \\ Center Schleswig-Holstein, \\ Department of Pediatrics, Kiel, \\ Germany (A Möricke MD); \\ Department of Pediatrics, Oulu University Hospital, Oulu, Finland (R Niinimäki MD); EORTC Children's Leukemia Group and University Department of Pediatric Oncology CHR Citadelle, Liège, Belgium (C Piette MD); Clinic of Pediatric Hematology \\ Oncology, Department of Women's and Children's \\ Health, Padova, Italy (M C Putti MD); University of Utah, Department of Pediatrics and Huntsman Cancer}

\section{Methods}

\section{Toxic effects considered by the PTWG}

Representatives from 15 PdL groups listed all acute toxic effects of childhood lymphoblastic leukaemia treatment that are serious but either too rare to be addressed comprehensively within any single acute lymphoblastic leukaemia group, or needed consensus definitions for reliable comparison of incidences and outcome (appendix p 22). After initial discussions, those representatives decided that the toxic effects that were almost universally reversible and sufficiently common to be investigated within a single acute lymphoblastic leukaemia group-such as mucositis, bone-marrow and immune suppression, febrile neutropenia, skin rashes, hyperglycaemia, and several transient organ failuresshould not be pursued by the PTWG. Among the remaining toxic effects, treatment-related mortality and invasive fungal infections (apartfrom Pneumocystisjirovecii

27 toxic effects considered by PTWG

13 excluded

2 already addressed by other groups

1 too affected by local logistics and resources

6 multiple and complex causes

4 already well defined and graded by the CTCAE with

definitions suitable for children with acute lymphoblastic leukaemia

14 toxic effects selected. An ad-hoc working group was established for each toxicity

Each ad-hoc working group developed and refined:

- The definition for their toxic effect

- A section on their toxic effect for a detailed supportive PTWG document*

$\downarrow$

First face-to-face plenary meeting, April 25, 2014, at the International BFM Annual

Meeting, Prague, Czech Republic

Ad-hoc working groups presented their findings in standardised presentations:

- How each acute lymphoblastic leukaemia protocol defined and captured toxic effects

- Emphasis on protocol diversities

- Implications of a toxic effect for subsequent acute lymphoblastic leukaemia treatment

-Weaknesses and challenges associated with present definitions

- Proposal for PTWG consensus definition

\section{$\checkmark$}

Second face-to-face plenary meeting, Dec 5, 2014, at the American Society of

Hematology Annual Meeting, San Francisco, CA, USA

Ad-hoc working groups presented their findings in standardised presentations:

- Discussion of each toxic effect definition. Focus on clarity and clinical applicability

- Discussion of first detailed PTWG document draft and the comments received

from PdL acute lymphoblastic leukaemia groups

- Strategies for treatment modification, capture of toxic effects, and registration were

not discussed further because the primary aim was to obtain consensus definitions

Third face-to-face plenary meeting, May 8,2015 , the International BFM Annual
Meeting, Budapest, Hungary
Ad-hoc working groups presented their findings in standardised presentations:
• Each toxic effect definition finalised
- Discussion and finalisation of second draft of detailed PTWG document

Figure: Delphi process to reach consensus definitions for 14 toxic effects

PTWG=Ponte di Legno toxicity working group. CTCAE=National Cancer Institute Common Terminology Criteria for Adverse Events. PdL=Ponte di Legno. BFM= Berlin-Frankfurt-Münster. *Versions of the detailed PTWG document were circulated to all PdL groups for comments during September, 2014, and March, 2015, and are now included as the appendix.

pneumonia) had been addressed, ${ }^{6}$ or are being addressed at present by other international working groups. Transferral to an intensive care unit was deemed to be too greatly affected by local logistics and resources to be included for consideration. Furthermore, the PTWG did not regard toxic effects that have multiple and complex causes (such as hepatic failure) as candidates for PTWG consensus definitions, although they might be relevant for future prospective registration to quantify and qualify the burden of antileukaemic treatment. Finally, the PTWG did not address several toxic effects that were serious but already defined and graded by the CTCAE with definitions suitable for children with acute lymphoblastic leukaemia.

\section{Formation of consensus definitions}

Since the pathogeneses and natural histories for most of the remaining 14 prioritised toxic effects are poorly understood from a biological point of view, the PTWG chose a Delphi process for obtaining expert opinionbased consensus definitions for these toxicities. ${ }^{7.8}$ The PTWG established an ad-hoc working group for each of the 14 toxicities, including a chair for each group, with initial representation of experts from at least three of the 15 involved acute lymphoblastic leukaemia groups, which were subsequently expanded after each face-to-face plenary PTWG meeting (appendix p 22). Each ad-hoc working group reviewed the present scientific literature on their toxic effect with a special focus on acute lymphoblastic leukaemia cohort publications, and reviewed the toxic effect sections of 13 treatment protocols currently used by PdL groups. Additionally, one author (MK) added information on the Japanese acute lymphoblastic leukaemia protocols because they were not available in English. Each ad-hoc working group's results and considerations were debated within their collaborative acute lymphoblastic leukaemia group and at three consecutive face-to-face plenary meetings (figure). These face-to-face discussions were open to other individuals from the PdL, even if they were not directly involved in developing the final toxic effect definitions. On the basis of discussions at these meetings and comments from the involved PdL acute lymphoblastic leukaemia groups, all groups finalised and approved the definitions of these 14 toxicities (panel).

\section{Findings}

Although the acute lymphoblastic leukaemia protocols all have sections on treatment-related toxic effects, none of the protocols assessed address all of the 14 toxic effects listed in this report. When addressed, however, they use various definitions (appendix p 24). Furthermore, although a specific toxic effect might be highlighted and described in detail by one acute lymphoblastic leukaemia treatment protocol (eg, posterior reversible encephalopathy syndrome), other protocols might not mention 
Panel: Consensus definitions and gradings of 14 acute toxic effects associated with treatment of childhood acute lymphoblastic leukaemia

\section{Hypersensitivity to asparaginase}

An adverse local or general response from exposure to asparaginase characterised by flushing, rash, urticaria, drug fever, dyspnoea, symptomatic bronchospasm, oedema or angio-oedema, hypotension, and/or anaphylaxis. Grading:

- Mild: transient flushing or rash, drug-induced fever $<38^{\circ} \mathrm{C}$.

- Severe: drug fever $\geq 38^{\circ} \mathrm{C}$; allergy-related oedema or angio-oedema; dyspnoea and/or symptomatic bronchospasm with or without urticaria; and/or hypotension and anaphylaxis with indication for asparaginase infusion interruption and parenteral medication (eg, antihistamines, glucocorticosteroids).

\section{Hyperlipidaemia}

Triglycerides/cholesterol blood concentrations greater than upper normal limit (UNL). Grading:

1 Mild: triglycerides/cholesterol $<10$ times UNL.

2 Moderate: triglycerides/cholesterol $10-20$ times UNL.

3 Severe: triglycerides/cholesterol $>20$ times UNL.

Routine measurements should be done only as part of research protocols. Dose modification based only on laboratory findings is not recommended.

\section{Osteonecrosis}

Osteonecrosis results from the temporary or permanent loss of the blood supply to the bones, which can cause pain, limitation in activity of daily living, and potentially the collapse of an articulating surface with enhanced pain and development of arthritis. The disorder should be confirmed by MRI. Grading:

1 Asymptomatic with findings only by MRI.

2 Symptomatic, not limiting or only slightly limiting self-care activity of daily living. Lesions only outside joint lines in non-weight-bearing bones.

3 Symptomatic, not limiting or only slightly limiting self-care activity of daily living. Lesions in weight-bearing bones or affecting joint lines in non-weight-bearing bones.

4 Symptomatic with deformation by imaging of one or more joints and/or substantially limiting self-care activity of daily living.

\section{Asparaginase-associated pancreatitis}

At least two of three features must be fulfilled: abdominal pain strongly suggestive of pancreatitis; serum lipase or amylase three or more times UNL; and characteristic imaging findings of pancreatitis (ultrasound, CT, or MRI). Re-exposure should only be considered in mild cases. Grading:

1 Mild: symptoms and enzyme elevations more than three times UNL that last less than $72 \mathrm{~h}$.

2 Severe: symptoms and/or enzyme elevations more than three times UNL that last more than $72 \mathrm{~h}$, or haemorrhagic pancreatitis, pancreatic abscess, or cyst.

3 Death from pancreatitis.

\section{Arterial hypertension}

Systolic blood pressure and/or diastolic blood pressure at or greater than the 95th percentile for sex, age, and height on three or more occasions (three consecutive days, or separate clinic visits if outpatient). Grading:

1 Systolic blood pressure/diastolic blood pressure in the 90th-95th percentile for age and/or blood pressure exceeding $120 / 80 \mathrm{~mm} \mathrm{Hg}$.

2 Recurrent or persistent systolic blood pressure/diastolic blood pressure greater than the 95th percentile for age at three separate measurements or lasting more than $72 \mathrm{~h}$ with monotherapy indicated.

3 Recurrent or persistent systolic blood pressure/diastolic blood pressure greater than 95th percentile for age at three separate measurements or lasting more than $72 \mathrm{~h}$ and needing more than one drug or additional intensive treatment than grade 2 for blood pressure control.

4 Life-threatening consequences (eg, hypertensive crisis with transient or permanent neurological deficit and urgent intervention needed)

5 Death from hypertension.

\section{Posterior reversible encephalopathy syndrome}

Posterior reversible encephalopathy syndrome is a clinical diagnosis based on any combination of transient headache, confusion, seizures, and visual disturbances in combination with characteristic, but transient, contrast-enhanced and diffusion-weighted imaging MRI findings. Diagnosis can be supported by electroencephalogram findings, occurrence during early months of treatment, and presence of arterial hypertension. No grading.

\section{Seizures}

A disorder characterised by sudden, involuntary skeletal muscle contractions of cerebral or brainstem origin. Grading:

1 Brief partial seizure.

2 Brief generalised seizure.

3 Multiple seizures despite medical intervention.

4 Life-threatening, prolonged, or repetitive seizures.

5 Death from seizures.

\section{Depressed level of consciousness}

Abnormal changes in level of arousal or altered content of a patient's thought processes.

Quantified by Glasgow Coma Scale or the patient being alert (appears wakeful and aware of self and environment), lethargic (mild reduction in alertness), obtunded (moderate reduction in alertness with increased response time to stimuli), stuporous (deep sleep; arousal only by vigorous or repetitive stimulation and return to deep sleep when discontinued), or comatose (unconscious, sleep-like appearance and behaviourally unresponsive to all external stimuli).
Institute, Salt Lake City, UT, USA (Prof E Raetz MD); Dana-Farber Cancer Institute and Boston Children's Hospital, Boston, MA, USA

(L B Silverman MD); Department of Paediatric and Adolescent Haematology/ Oncology, and Children's Haemopoietic Stem Cell Transplant Unit, Great North Children's Hospital, Newcastle upon Tyne, UK

(Prof R Skinner MD):

Department of Pediatrics, Aalborg University Hospital,

Aalborg, Denmark

(RTuckuviene MD); Dutch Childhood Oncology Group The Hague, Netherlands (I van der Sluis MD); Erasmus Medical Center, Sophia Children's Hospital, Department of Pediatric Hematology-Oncology, Rotterdam, Netherlands (I van der Sluis); and University Hospital Motol, Department of Pediatric

Hematology/Oncology, Prague, Czech Republic (E Zapotocka MD)

Correspondence to: Prof Kjeld Schmiegelow, Department of Pediatrics and Adolescent Medicine, Rigshospitalet, 2100 Copenhagen, Denmark kschmiegelow@rh.dk See Online for appendix 
(Panel continued from previous page)

Can involve simple capabilities (speech, calculations, or spelling) and more complex modalities (emotions, behaviour, or personality) with confusion, disorientation, hallucinations, poor comprehension, or verbal expressive difficulty.

\section{Methotrexate-related stroke-like syndrome}

Neurotoxicity occurring within 21 days of intravenous or intrathecal methotrexate with three characteristics that all need to be fulfilled:

- New onset of one or more of paresis or paralysis; movement disorder or bilateral weakness; aphasia or dysarthria; altered mental status including consciousness (eg, somnolence, confusion, disorientation, and emotional lability); and/or seizures with at least one of the other symptoms.

- Either characteristic, but often transient, white matter changes indicating leukoencephalopathy on MRI or a characteristic clinical course with waxing and waning symptoms usually leading to complete (sometimes partial) resolution within a week.

- No other identifiable cause.

Characteristic oval-shaped lesions of the subcortical white matter (mostly frontal or parietal) on MRI are best seen on diffusion-weighted (hyperintense) or apparent diffusion coefficient (hypointense) images. Can be graded 1-5 according to CTCAEv4.03 for encephalopathy.

\section{Peripheral neuropathy}

Peripheral motor or sensory neuropathy, including pain and constipation, due to inflammation or degeneration of the peripheral motor or sensory nerves. Grading:

1 Loss of deep tendon reflexes, slight paraesthesia, numbness, or pain that does not limit instrumental activity of daily living or require treatment.

2 Moderate symptoms that somewhat limit instrumental activity of daily living (eg, alters fine motor skills such as buttoning a shirt) and/or paraesthesia, numbness, or pain that are controllable by non-narcotic medications.

3 Severe symptoms limiting self-care activity of daily living, including gait impairment, inability to perform fine motor tasks; and/or paraesthesia, numbness, or pain that require narcotics.

4 Complete paralysis or life-threatening consequences (eg, vocal cord paralysis) with urgent need for intervention, or severe pain that is not controlled by narcotics.

5 Death from peripheral neuropathy (eg, vocal cord paralysis).
High-dose methotrexate-related severe nephrotoxicity Increase in plasma creatinine of more than $0.3 \mathrm{mg} / \mathrm{dL}$ ( $26.5 \mu \mathrm{mol} / \mathrm{L}$ ) and/or a relative increase of 1.5 times greater than a baseline value (measured within 4 days prior to hydration preceding high-dose methotrexate) together with plasma methotrexate concentrations at one or more timepoints after initiation of the methotrexate infusion: $36 \mathrm{~h}$ methotrexate more than $20 \mu \mathrm{mol} / \mathrm{L}, 42 \mathrm{~h}$ methotrexate more than $10 \mu \mathrm{mol} / \mathrm{L}$, and/or $48 \mathrm{~h}$ methotrexate more than $5 \mu \mathrm{mol} / \mathrm{L}$. Renal toxic effects can be graded according to CTCAEv4.03.

\section{Sinusoidal obstruction syndrome}

Fulfilment of at least three of five, otherwise unexplained, criteria: hepatomegaly; hyperbilirubinaemia more than UNL; ascites; weight gain of at least $5 \%$; and thrombocytopenia (transfusion-resistant and/or otherwise unexplained by treatment [eg, myelosuppression]). Doppler ultrasound could document changes in hepatic portal venous flow and rule out alternative causes, but normal findings do not exclude sinusoidal obstruction syndrome. Grading:

1 Mild: bilirubin less than $103 \mu \mathrm{mol} / \mathrm{L}$ and weight gain less than $5 \%$.

2 Moderate: bilirubin 103-342 $\mu \mathrm{mol} / \mathrm{L}$ and/or weight gain more than $5 \%$ or ascites.

3 Severe: bilirubin more than $342 \mu \mathrm{mol} / \mathrm{L}$ and/or respiratory or renal failure or hepatic encephalopathy.

4 Death due to sinusoidal syndrome.

\section{Thromboembolism}

Venous and/or arterial thromboembolism. Confirmation by imaging (ultrasonography, computed tomography, magnetic resonance imaging) or by autopsy is required for grade 2 and higher. Grading:

1 Superficial thrombophlebitis or central venous line-associated deep venous thrombosis without symptoms (eg, pain or shortness of breath) or objective signs (eg, swelling, discolouration, or collaterals); or causing only central venous line dysfunction. Systemic anticoagulation not given.

2A Asymptomatic thromboembolism (including asymptomatic cerebral thrombosis). Systemic anticoagulation is usually given (not evidence-based).

2B Symptomatic deep vein thrombosis, systemic anticoagulation indicated.

(Continued on next page) that toxic effect. Many protocols agree on individual toxic effect definitions and grading, especially when the CTCAE is applied, ${ }^{5}$ but no two protocols share identical definitions of all toxic effects, and no toxic effect definition is shared by all protocols. Some protocols request data capture of any grade of a toxic effect, whereas other protocols only address the most severe grades. Additionally, the consequence of a specific toxic effect occurring varies by protocol, from complete withdrawal of an antileukaemic drug (eg, asparaginase after pancreatitis) to no consequences, including the acceptance of re-exposure, although this is not always specifically stated. All details of consensus toxicity definitions, including background, guidelines, and considerations before toxicity definition, can be found in the appendix. 
(Panel continued from previous page)

3 Symptomatic pulmonary embolism, cardiac mural thrombus without cardiovascular compromise, symptomatic cerebral sinovenous thrombosis, or arterial ischaemic stroke; all require systemic anticoagulation/antiaggregation.

4 Life-threatening thromboembolism, including arterial insufficiency, haemodynamic or neurological instability. Urgent intervention needed.

5 Death due to thromboembolism.

Pneumocystis jirovecii pneumonia

- Confirmed Pjirovecii pneumonia: presence of P jirovecii organisms from a patient with fever, abnormal chest radiograph compatible with $P$ jirovecii infection, and/or hypoxaemia.
- Probable Pjirovecii pneumonia: pneumonia of undetermined origin (fever, P jirovecii pneumonia compatible chest radiograph, and/or hypoxaemia) and responding to empirical treatment with co-trimoxazole.

Presence of $P$ jirovecii organisms identified through cytological examination (Gomori-Grocott or Gram-Weigert staining), P jirovecii-specific PCR, or P jirovecii immunofluorescence in a lung sample (bronchoalveolar lavage, bronchial aspiration, transbronchial biopsy, transthoracic needle aspiration, lung biopsy, or sputum).

CTCAEv4.03=National Cancer Institute Common Terminology Criteria for adverse events version 4.03 .

\section{Hypersensitivity to asparaginase}

Allergic reactions to asparaginase are frequent.,10 All protocols address hypersensitivity, but only a few address silent inactivation (ie, neutralising antibodies with reduced enzymatic activity), and then with various definitions of trough levels and timepoints for measurements, and none address allergic-like reactions (eg, vomiting, stomach ache, or rash) without inactivation of asparaginase or indications for change in treatment (appendix p 2). The PTWG reached consensus that definitions of hypersensitivity, silent inactivation, and allergy-like reactions were needed, although each could pose practical clinical challenges. All but one group use pegylated asparaginase as front-line treatment. Because pegylated asparaginase becomes inactivated in virtually all patients with an allergic reaction irrespective of its severity, ${ }^{9}$ any degree of hypersensitivity should logically lead to a change from Escherichia coli-derived pegylated asparaginase to Erwinia chrysanthemi-derived asparaginase. $^{10}$ In addition to the definition of asparaginase hypersensitivity, the PTWG defined silent inactivation in patients without clinical allergy as trough asparaginase activity levels less than the lower level of quantification (LLQ; preferably measured in two independent samples)—ie, a day 7 asparaginase activity level of less than 100 international units per L or a day 14 level of less than LLQ in case of biweekly pegylated asparaginase, or both; and a $48 \mathrm{~h}$ post-dose level of less than LLQ in case of E chrysanthemi-derived asparaginase (given two to three times a week).

\section{Hyperlipidaemia}

Both asparaginase and glucocorticosteroids can cause transient and occasionally severe hypertriglyceridaemia. This disorder could lead to toxic complications (eg, thrombosis and cardiovascular late effects) although these complications are so far poorly documented..$^{11}$ However, only a few protocols address this toxic effect, mostly without a clear definition, and only one protocol recommends routine monitoring of serum triglycerides for selected patients. The PTWG reached consensus on defining severity of hypertriglyceridaemia on the basis of levels, and also that routine monitoring should only take place as part of a research strategy.

\section{Osteonecrosis}

Osteonecrosis is a very common side-effect of acute lymphoblastic leukaemia treatment that is addressed by all protocols, although each has diverse definitions ${ }^{12}$ and differing guidelines for further glucocorticosteroid treatment. $^{12}$ None of the protocols clarify the role, interpretation, or classification of imaging. ${ }^{13}$ All but one protocol used the CTCAE for clinical grading. The PTWG reached consensus that MRI should be applied for confirmation of clinically symptomatic disease rather than for screening patients, and that MRI should only be used for screening within a research project.

\section{Asparaginase-associated pancreatitis}

Asparaginase-associated pancreatitis has low direct mortality, but is one of the most frequent causes of discontinuation of asparaginase treatment, which could increase risk of relapse. ${ }^{14}$ All but one protocol provides a definition of asparaginase-associated pancreatitis, with grading using either the CTCAE criteria or some modification of the Atlanta criteria ${ }^{15}$ (ie, abdominal pain suggestive of acute pancreatitis; serum amylase or serum lipase, or both, at or more than 2-3 times upper normal limit; and imaging findings characteristic of acute pancreatitis) although with variation as to whether two or three criteria should be fulfilled. Some protocols recommended measurements of both amylase and lipase because a lipase measurement is more specific and sensitive than an amylase measurement. Protocols with extended use of asparaginase generally recommend truncation of asparaginase treatment only in cases of severe asparaginase-associated pancreatitis. 


\section{Arterial hypertension}

Arterial hypertension is common, especially during the first months of antileukaemic treatment. ${ }^{16}$ However, none of the protocols address arterial hypertension as an isolated toxic effect, instead mentioning it only in association with posterior reversible encephalopathy syndrome. The PTWG reached consensus that the American Academy of Pediatrics ${ }^{17}$ and the CTCAE guidelines should provide classifications of hypertension that are applicable to its transient occurrence during acute lymphoblastic leukaemia treatment.

\section{Posterior reversible encephalopathy syndrome}

Although posterior reversible encephalopathy syndrome is a clinicoradiological entity that is frequently reported during the first months of acute lymphoblastic leukaemia treatment, reflecting disturbances of cerebrovascular autoregulation, and is inconsistently characterised by headache, altered mental status, seizures, and visual disturbances, ${ }^{18}$ seven protocols do not address it at all, and only one addresses it in detail. When addressed, protocols apply the CTCAE grading used for any encephalopathy, despite its restricted usefulness for posterior reversible encephalopathy syndrome. Except for postponing intrathecal treatment until symptoms resolve, no antileukaemic treatment modifications are recommended in any of the protocols.

\section{Seizures}

Seizures occur in about $10 \%$ of children with acute lymphoblastic leukaemia. ${ }^{19}$ Most acute lymphoblastic leukaemia protocols grade seizures clinically according to CTCAE grading, which does not require electroencephalography and excludes absence seizures, which are rare in childhood acute lymphoblastic leukaemia. Seizures can occur both as an isolated symptom, together with various other toxic effects of the CNS (eg, intracranial haemorrhage or thrombosis, posterior reversible encephalopathy syndrome, and methotrexate related stroke-like syndrome), and second to infections and electrolyte and metabolic disturbances. The PTWG decided not to include causation in the definition, but will address this complexity in the registration of seizures as a toxicity in the future.

\section{Depressed level of consciousness}

The protocols provide grading for encephalopathy in general, but not specifically in the context of decreased consciousness or even coma, potentially reflecting the complexity of both classification of the toxic effect itself and its multiple causes such as infection, altered body temperature, electrolyte and metabolic disturbances, vascular or neurological complications, and direct toxic effects of chemotherapy. The PTWG consensus definition is based on clinical findings only.

\section{Methotrexate-related stroke-like syndrome}

Methotrexate-related stroke-like syndrome, which is characterised by focal neurological deficits or hemiparesis, and often accompanied by disturbances in speech, or all three, often develops within 2 to 3 weeks after methotrexate administration, and can last hours to days during which symptoms can wax and wane..$^{20,2}$ All but one protocol provided grading for encephalopathy, although not specifically for methotrexate-related stroke-like syndrome, and used the CTCAE or the US Eastern Cooperative Oncology Group criteria. Only five protocols address this syndrome, of which only one describes the characteristic symptoms in detail, and only a few providing (various) guidelines for methotrexate re-exposure once the methotrexate-related neurotoxicity has resolved. Although MRI will not always be able to confirm methotrexate-related stroke-like syndrome, it is included in the consensus definition because of the characteristic changes it often shows, and its ability to distinguish between methotrexate-related stroke-like syndrome and posterior reversible encephalopathy syndrome.

\section{Peripheral neuropathy}

Peripheral motor or sensory neuropathy, or both, are common and are generally caused by vincristine (in which case they are nearly always reversible). ${ }^{22}$ No protocols recommend discontinuation of vincristine except in cases of paralysis (occasionally caused by Charcot-Marie-Tooth disease), but several protocols recommend dose reduction in CTCAE grade 3-4 cases of paraesthesia or motor paralysis. This toxic effect is addressed by all treatment protocols with the CTCAE grading, except for one group applying the Balis scale. ${ }^{23}$ The PTWG agreed to use the CTCAE grading with minor modifications.

\section{High-dose methotrexate-related nephrotoxicity}

All protocols that include administration of high-dose methotrexate $\left(2 \cdot 5-5 \cdot 0 \mathrm{~g} / \mathrm{m}^{2}\right)$ have clear, although diverse, guidelines for hydration, alkalinisation, and folinic acid rescue. The protocols differ in their definition of delayed methotrexate elimination both with respect to methotrexate concentrations and timepoints from initiation of the methotrexate infusion. In cases of severely delayed elimination of methotrexate, less than half of the protocols include guidelines for the use of carboxypeptidase that enzymatically breaks down methotrexate to non-toxic metabolites. ${ }^{24}$ Because of the very strong association between renal impairment and delayed methotrexate clearance, both parameters were included in the consensus definition.

\section{Sinusoidal obstructive syndrome}

Sinusoidal obstructive syndrome or veno-occlusive disease is most commonly seen after haemopoietic stem cell transplantation and during 6-thioguanine containing maintenance treatment, but rarely with 
6-mercaptopurine-based maintenance treatment. ${ }^{25}$ Although the general risks of hyperbilirubinaemia and elevations of aminotransferases during acute lymphoblastic leukaemia treatment are mentioned in most of the protocols, sinusoidal obstructive syndrome is not included in the CTCAE, and only two of the protocols specifically address the syndrome, with only one including a definition. The PTWG consensus definition is based on the combinations of at least three of five clinical findings and does not require imaging, although imaging might be of diagnostic benefit in selected cases.

\section{Thromboembolism}

Most of the protocols address thromboembolic events, with all protocols grading them according to the CTCAE, but varying with respect to which grades are to be reported as severe adverse events. In cases of thromboembolism during asparaginase treatment, all six protocols that address the issue recommend re-exposure with asparaginase once the patient's clinical condition has stabilised and low molecular weight heparin has been instituted. ${ }^{26}$ The PTWG consensus definition of thromboembolism incorporates both localisation and severity of symptoms.

\section{Pjirovecii pneumonia}

The high risk of $P$ jirovecii pneumonia when prophylaxis for the infection is not given during treatment of childhood acute lymphoblastic leukaemia is recognised in all protocols, but they differ in their prescribed dose of prophylactic co-trimoxazole and in the required diagnostic criteria. ${ }^{27}$ The PTWG consensus definition distinguishes between confirmed and probable $P$ jirovecii pneumonia.

\section{Discussion}

In childhood acute lymphoblastic leukaemia, the term event-free survival traditionally encompasses five clearcut events, namely: death during induction; resistance to first-line treatment; relapse of acute lymphoblastic leukaemia; non-leukaemic death during clinical remission; and development of a second cancer. ${ }^{1}$ Although this composite measure of treatment outcome seemed sufficient when life expectancy for children with acute lymphoblastic leukaemia was poor, it falls short of present needs. Although many patients with a late relapse or a second cancer have a fair chance of being cured by second-line treatment, we cannot currently reverse their chronic toxic effects. Each year, thousands of children around the world are cured after treatment for acute lymphoblastic leukaemia. However, many of them will have been burdened by severe acute toxic effects that can cause permanent organ damage, such as osteonecrosis, chronic pancreatitis, thrombosis, and neurotoxicity, and even more patients will develop additional severe late effects that challenge their ability to establish and live a normal adult life..$^{28,29}$
Although the cumulative risk of each of the 14 acute toxic effects addressed in this report is about $5-10 \%$ or less, about half of all patients will be affected by at least one of the 14 effects..$^{30}$ As such, in the overall evaluation of acute lymphoblastic leukaemia treatment protocols, there is a need for the development of strategies to quantify the overall acute and long-term burden of treatment and balance it against event-free survival. These strategies will require uniform reporting in trials of acute lymphoblastic leukaemia of both life-threatening and fatal toxic effects and of toxic effects that are associated with substantial late effects.

The development of evidence-based preventive interventions for the toxic effects of acute lymphoblastic leukaemia treatment require consensus definitions of toxic effects to compare outcome across protocols; common strategies for capture and registration of toxic effects; and international collaboration to identify host genome variants and exposures (eg, antileukaemic treatment, co-medication, and food-drug interaction) associated with the risk of specific toxic effects. Not all toxic effect definitions presented in this Review are clear-cut, which mainly reflects their uncertain pathophysiology. Furthermore, several toxic effects can have overlapping symptoms (such as from the CNS), making precise classification challenging. Additionally, guidelines for interventions can be directed towards the symptom (eg, seizures or hypertension due to posterior reversible encephalopathy syndrome) or the underlying pathology (eg, methotrexate-related stroke-like syndrome). Accordingly, future registrations of some organ toxic effects should allow entry of both separate symptoms (eg, seizures) and a putative syndrome (eg, posterior reversible encephalopathy syndrome).

We developed the toxic effect definitions listed in this report after reviewing the existing scientific literature and current acute lymphoblastic leukaemia protocols, and using the Delphi method to develop expert consensus definitions. ${ }^{7.8}$ These definitions are a starting point for developing evidence-based guidelines regarding optimum management and prevention strategies. Although the definitions are supported by the PdL acute lymphoblastic leukaemia groups and should be widely applicable, their clinical and biological validity will emerge in parallel with their implementation, and a deeper understanding of the pathogenesis of the toxic effects gained by relevant in-vitro and animal models and international research collaboration.

The present CTCAE criteria for toxic effects are mostly clinical and their grading is generally based on a five grade scale. The CTCAE criteria benefit from their long history of use, and from the standardisation of number of grades and uniformity of definitions. However, they are not specifically adapted to the administered anticancer treatment and some toxic effects (eg, osteonecrosis and infertility) are never life-threatening or fatal, thus reducing the number of grades. Furthermore, the 
inclusion of medical intervention in several classifications is controversial because it suggests that intervention is needed for a specific grade of toxic effect. Additionally, the definition might also reclassify a patient if a decision is made to refrain from an intervention because of local practice or patient preference rather than just the severity of the toxic effect. Finally, the definition of toxic effect grades should also be coherent with re-exposure guidelines for acute lymphoblastic leukaemia treatment (eg, asparaginase in cases of mild asparaginase-associated pancreatitis).

Because of national regulations, some trial groups will be mandated to continue to register toxic effects according to specific guidelines, such as the CTCAE (currently under revision) ${ }^{5}$ in the USA. These acute lymphoblastic leukaemia groups will need to consider toxic effect capture and registration strategies that cover both systems to allow future reporting of their data in a format that allows reliable comparison of the toxic effect profile with acute lymphoblastic leukaemia groups that use the PdL toxic effect definitions.

The subsequent, but equally challenging, goal for the PTWG is now to develop common strategies for toxic effect capture and registration because targeting selected toxic effects could favour their capture at the expense of non-targeted, but routinely registered, CTCAE-graded toxic effects, even though the non-targeted toxic effects might be equally important clinically. ${ }^{31}$ Additionally, the PTWG will address guidelines for drug re-exposures, explore the effect of host genome variants on toxic effect risks, and develop consensus algorithms that balance toxicity and efficacy in composite assessments of the outcome of acute lymphoblastic leukaemia treatment. As such, although many toxic effects that emerge when treating acute lymphoblastic leukaemia during childhood can be far more difficult to capture than the classic five treatment failures, they are just as crucial to include in future intervention trials to improve the outcome of children with acute lymphoblastic leukaemia.

\section{Contributors}

All authors contributed equally to the establishment of the PTWG and sharing protocols, including the toxic effects sections. Authors are either representing their collaborative acute lymphoblastic leukaemia group or have chaired an ad-hoc toxicity working group under the PTWG, or both. KS chaired the PTWG and coordinated this report. All authors contributed to the data collection and interpretation. KS drafted the first version of the manuscript, which was subsequently revised and approved by all authors.

Declaration of interests

We declare no competing interests.

References

1 Pui CH, Yang JJ, Hunger SP, et al. Childhood acute lymphoblastic leukemia: progress through collaboration. J Clin Oncol 2015; 33: 2938-48.

2 Oeffinger KC, Mertens AC, Sklar CA, et al. Chronic health conditions in adult survivors of childhood cancer. $N$ Engl J Med 2006; 355: 1572-82.

3 Essig S, Li QZ, Chen Y, et al. Risk of late effects of treatment in children newly diagnosed with standard-risk acute lymphoblastic leukaemia: a report from the Childhood Cancer Survivor Study cohort. Lancet Oncol 2014; 15: 841-51.
4 Moriyama T, Relling MV, Yang JJ. Inherited genetic variation in childhood acute lymphoblastic leukemia. Blood 2015; 125: 3988-95.

5 US Department of Health and Human Services. Common Terminology Criteria for Adverse Events (CTCAE). Version 4.0. May 28, 2009. http://evs.nci.nih.gov/ftp1/CTCAE/ CTCAE_4.03_2010-06-14_QuickReference_5x7.pdf (accessed Nov 4, 2015).

6 Alexander S, Pole JD, Gibson P, et al. Development of a reliable and valid system for classifying treatment-related mortality in children with cancer. Lancet Oncol 2015; 16: e604-10.

7 Zafar SY, Currow DC, Cherny N, Strasser F, Fowler R, Abernethy AP. Consensus-based standards for best supportive care in clinical trials in advanced cancer. Lancet Oncol 2012; 13: e77-82.

8 Powell C. The Delphi technique: myths and realities. J Adv Nurs 2003; 41: 376-82.

9 Tong WH, Pieters R, Kaspers GJL, et al. A prospective study on drug monitoring of PEGasparaginase and Erwinia asparaginase and asparaginase antibodies in pediatric acute lymphoblastic leukemia. Blood 2014; 123: 2026-33.

10 Ko RH, Jones TL, Radvinsky D, et al. Allergic reactions and antiasparaginase antibodies in children with high-risk acute lymphoblastic leukemia: a children's oncology group report. Cancer 2015; 121: 4205-11.

11 Niinimaki T, Harila-Saari A, Niinimaki R. The diagnosis and classification of osteonecrosis in patients with childhood leukemia. Pediatr Blood Cancer 2015; 62: 198-203.

12 Mattano LA, Jr, Devidas M, Nachman JB, et al. Effect of alternate-week versus continuous dexamethasone scheduling on the risk of osteonecrosis in paediatric patients with acute lymphoblastic leukaemia: results from the CCG-1961 randomised cohort trial. Lancet Oncol 2012; 13: 906-15.

13 Niinimaki T, Niinimaki J, Halonen J, Hanninen P, Harila-Saari A Niinimaki R. The classification of osteonecrosis in patients with cancer: validation of a new radiological classification system. Clin Radiol 2015; 70: 1439-44.

14 Raja RA, Schmiegelow K, Frandsen TL. Asparaginase-associated pancreatitis in children. Br J Haematol 2012; 159: 18-27.

15 Banks PA, Bollen TL, Dervenis C, et al, Acute Pancreatitis Classification Working Group. Classification of acute pancreatitis 2012: revision of the Atlanta classification and definitions by international consensus. Gut 2013; 62: 102-111.

16 Kamdem LK, Hamilton L, Cheng C, et al. Genetic predictors of glucocorticoid-induced hypertension in children with acute lymphoblastic leukemia. Pharmacogenet Genomics 2008; 18: 507-14.

17 US Department of Health and Human Services. The fourth report on the diagnosis, evaluation, and treatment of high blood pressure in children and adolescents. http://www.nhlbi.nih.gov/files/docs/ resources/heart/hbp_ped.pdf. (accessed Nov 4, 2015).

18 de Laat P, Te Winkel ML, Devos AS, et al. Posterior reversible encephalopathy syndrome in childhood cancer. Ann Oncol 2011; 22: 472-78.

19 Ochs JJ, Bowman WP, Pui CH, Abromowitch M, Mason C, Simone JV. Seizures in childhood lymphoblastic leukaemia patients. Lancet 1984; 2: 1422-24.

20 Rubnitz JE, Relling MV, Harrison PL, et al. Transient encephalopathy following high-dose methotrexate treatment in childhood acute lymphoblastic leukemia. Leukemia 1998; 12: 1176-81.

21 Bond J, Hough R, Moppett J, Vora A, Mitchell C, Goulden N. 'Stroke-like syndrome' caused by intrathecal methotrexate in patients treated during the UKALL 2003 trial. Leukemia 2013; 27: 954-56.

22 Diouf B, Crews KR, Lew G, et al. Association of an inherited genetic variant with vincristine-related peripheral neuropathy in children with acute lymphoblastic leukemia. JAMA 2015; 313: 815-23.

23 Lavoie Smith EM, Li L, Hutchinson RJ, et al. Measuring vincristine-induced peripheral neuropathy in children with acute lymphoblastic leukemia. Cancer Nurs 2013; 36: E49-60.

24 Widemann BC, Schwartz S, Jayaprakash N, et al. Efficacy of glucarpidase (carboxypeptidase g2) in patients with acute kidney injury after high-dose methotrexate therapy. Pharmacotherapy 2014; 34: 427-39.

25 Escherich G, Richards S, Stork LC, Vora AJ, Childhood Acute Lymphoblastic Leukaemia Collaborative G. Meta-analysis of randomised trials comparing thiopurines in childhood acute lymphoblastic leukaemia. Leukemia 2011; 25: 953-59. 
26 Grace RF, Dahlberg SE, Neuberg D, et al. The frequency and management of asparaginase-related thrombosis in paediatric and adult patients with acute lymphoblastic leukaemia treated on Dana-Farber Cancer Institute consortium protocols. Br J Haematol 2011; 152: 452-59.

27 Levinsen M, Shabaneh D, Bohnstedt C, et al. Pneumocystis jiroveci pneumonia prophylaxis during maintenance therapy influences methotrexate/6-mercaptopurine dosing but not event-free survival for childhood acute lymphoblastic leukemia. Eur J Haematol 2012; 88: 78-86.

28 Lund LW, Winther JF, Dalton SO, et al. Hospital contact for mental disorders in survivors of childhood cancer and their siblings in Denmark: a population-based cohort study. Lancet Oncol 2013; 14: $971-80$.
29 Licht SD, Winther JF, Gudmundsdottir T, et al. Hospital contacts for endocrine disorders in adult life after childhood cancer in Scandinavia (ALiCCS): a population-based cohort study. Lancet 2014; 383: 1981-89.

30 Frandsen TL, Heyman M, Abrahamsson J, et al. Complying with the European Clinical Trials directive while surviving the administrative pressure-an alternative approach to toxicity registration in a cancer trial. Eur J Cancer 2014; 50: 251-59.

31 Miller TP, Li Y, Kavcic M, et al. Accuracy of adverse event ascertainment in clinical trials for pediatric acute myeloid leukemia. J Clin Oncol 2016; 34: 1537-43. 